\title{
The Evaluation of the Impact of New Technologies for Different Powertrain Medium-Duty Trucks on Fuel Consumption
}

2016-01-8134

Published 09/27/2016

\section{Lijuan Wang, Adam Duran, Kenneth Kelly, Arnaud Konan, Michael Lammert, and Robert}

\section{Prohaska}

National Renewable Energy Laboratory

CITATION: Wang, L., Duran, A., Kelly, K., Koana, A. et al., "The Evaluation of the Impact of New Technologies for Different Powertrain Medium-Duty Trucks on Fuel Consumption," SAE Technical Paper 2016-01-8134, 2016, doi:10.4271/2016-01-8134.

Copyright (C) 2016 SAE International

\begin{abstract}
In this paper, researchers at the National Renewable Energy Laboratory present the results of simulation studies to evaluate potential fuel savings as a result of improvements to vehicle rolling resistance, coefficient of drag, and vehicle weight as well as hybridization for four powertrains for medium-duty parcel delivery vehicles. The vehicles will be modeled and simulated over 1,290 real-world driving trips to determine the fuel savings potential based on improvements to each technology and to identify best use cases for each platform. The results of impacts of new technologies on fuel saving will be presented, and the most favorable driving routes on which to adopt them will be explored.
\end{abstract}

\section{Introduction}

This study explores modeling the fuel savings potentials of a variety of vehicle optimization scenarios across several powertrains in a medium-duty (MD) parcel delivery vehicle application. Vehicle modeling was performed using real-world data captured from hundreds of vehicles operating across the United States, totaling in excess of 1,000 days of operation. The real-world data used in this project was obtained from several geographic locations, including California, Minnesota, Arizona, Maryland, and Texas, in partnership with parcel delivery industry leaders. These drive cycles were used to explore the benefits of reductions in rolling resistance, aerodynamic drag, and vehicle mass for typical class 5/6 step vans such as is shown in Figure 1.

The National Renewable Energy Laboratory's (NREL's) Future Automotive Systems Technology Simulator (FASTSim), a high-level advanced vehicle powertrain systems analysis tool supported by the U.S. Department of Energy's Vehicle Technologies Office was selected as the modeling platform for use in this study [1]. FASTSim was chosen over alternative modeling options due to its quick and simple approach for comparing powertrains and for its ability to perform large-scale batch simulations in a time-efficient manner.

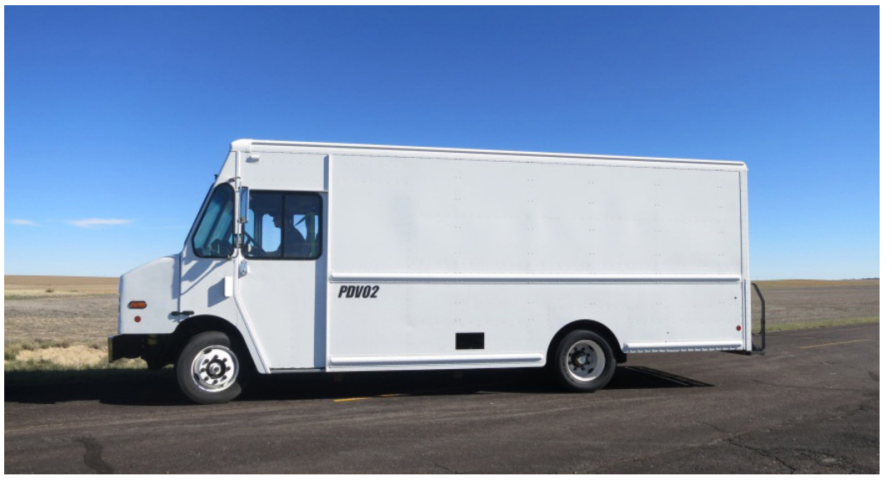

Figure 1. Example of step van

\section{The FASTSim Vehicle Model}

The MD trucks used in this research were a diesel conventional parcel delivery vehicle, a gasoline conventional parcel delivery vehicle, a diesel hybrid electric vehicle (HEV), and a diesel hydraulic electric vehicle (HHV). Specifications used as the basis for FASTSim modeling were sourced from NREL's Renewable Fuels and Lubricants (ReFUEL) Laboratory data captured during previous NREL field testing activities $[\underline{2}, \underline{3}, \underline{4}, \underline{5}, \underline{6}, \underline{7}, \underline{8}, \underline{9}]$. Table 1 details the key specifications for the four powertrain types examined in this study.

Table 1. Specifications of the four MD trucks

\begin{tabular}{|l|l|l|l|l|l|}
\hline & $\begin{array}{l}\text { Engine } \\
(\mathrm{kW})\end{array}$ & $\begin{array}{l}\text { Weight } \\
(\mathrm{kg})\end{array}$ & $\begin{array}{l}\text { Coeff. of } \\
\text { Rolling } \\
\text { Resistance }\end{array}$ & $\begin{array}{l}\text { Coeff. of } \\
\text { Aero- } \\
\text { dynamic } \\
\text { Drag }\end{array}$ & $\begin{array}{l}\text { Frontal } \\
\text { Aero- } \\
\text { dynamic } \\
\text { Area }\left(\mathrm{m}^{2}\right)\end{array}$ \\
\hline Diesel Conv. & 149 & 6,990 & 0.0071 & 0.71 & 6 \\
\hline $\begin{array}{l}\text { Gasoline } \\
\text { Conv. }\end{array}$ & 223 & 6,423 & 0.0092 & 0.70 & 6 \\
\hline Diesel HHV & 209 & 8,171 & 0.0092 & 0.70 & 6 \\
\hline Diesel HEV & 149 & 7,375 & 0.0092 & 0.70 & 6 \\
\hline
\end{tabular}


In characterizing the fuel consumption from MD vehicles, it is essential that the vehicles are tested or simulated under typical in-use behavior. In this study, several cycles covering a wide range of driving conditions were used. The vehicles were tested over the New York Composite (NY Comp); Heavy Heavy-Duty Diesel Truck (HHDDT); City Suburban Heavy Vehicle Cycle (CSHVC); Hybrid Truck Utility Forum Class 4 (HTUF); and Baltimore Custom Cycle (BCC), an NREL custom cycle that includes very aggressive driving behavior representative of real-world parcel delivery vehicle driving behavior in Baltimore, on the chassis dynamometer at the ReFUEL Laboratory to provide continuous fuel rate data. Table 2 shows the characteristics of the standard driving cycles, as well as the custom BCC. The detailed driving information can also be found in our previous research [10]. The parcel delivery trucks were modeled in FASTSim. The truck models were then calibrated by comparing the total fuel consumption (FC) from the chassis dynamometer test and the FASTSim simulation. The relative errors achieved for diesel trucks were all within $5.25 \%$. The results showed that the truck models were sufficiently calibrated and could be used as the basis for future parametric studies. The validation results are summarized in Table 3 .

Table 2. Driving cycle characteristics

\begin{tabular}{|l|l|l|l|l|}
\hline Cycle & $\begin{array}{l}\text { Kinetic } \\
\text { Intensity } \\
(1 / \text { mile })\end{array}$ & $\begin{array}{l}\text { Average } \\
\text { Speed } \\
(\mathrm{mph})\end{array}$ & $\begin{array}{l}\text { Stops per } \\
\text { Mile }\end{array}$ & $\begin{array}{l}\text { Distance } \\
(\text { miles })\end{array}$ \\
\hline NY COMP & 8.29 & 11.4 & 8.49 & 1.18 \\
\hline HHDDT & 0.17 & 35.59 & 1.63 & 26.05 \\
\hline CSHVC & 2.38 & 14.15 & 1.95 & 6.68 \\
\hline BCC & 1.35 & 22.57 & 1.37 & 20.42 \\
\hline HTUF & 1.51 & 22.49 & 2.51 & 11.17 \\
\hline
\end{tabular}

Table 3. Summaries of test and validation results

\begin{tabular}{|l|l|l|l|l|}
\hline \multirow{4}{*}{$\begin{array}{l}\text { Diesel } \\
\text { Conv. }\end{array}$} & Cycle & $\begin{array}{l}\text { ReFUEL } \\
\text { Lab (mpg) }\end{array}$ & $\begin{array}{l}\text { FASTSim } \\
(\mathrm{mpg})\end{array}$ & $\begin{array}{l}\text { Difference } \\
(\%)\end{array}$ \\
\cline { 2 - 5 } & HHDDT & 11.47 & 10.89 & -5.06 \\
\cline { 2 - 5 } & CSHVC & 9.48 & 9.46 & -0.21 \\
\cline { 2 - 5 } & BCC & 8.52 & 8.44 & -1.02 \\
\hline \multirow{4}{*}{$\begin{array}{l}\text { Gasoline } \\
\text { Conv. }\end{array}$} & NY COMP & 5.77 & 5.36 & -7.26 \\
\cline { 2 - 5 } & HHDDT & 9.18 & 8.74 & -4.78 \\
\cline { 2 - 5 } & CSHVC & 7.85 & 7.32 & -6.75 \\
\cline { 2 - 5 } & BCC & 6.54 & 6.97 & 6.53 \\
\hline \multirow{5}{*}{ Diesel HHV } & NY COMP & 10.84 & 11.13 & 2.67 \\
\cline { 2 - 5 } & HHDDT & 11.28 & 11.46 & 1.60 \\
\cline { 2 - 5 } & CSHVC & 12.82 & 12.28 & -4.24 \\
\cline { 2 - 5 } & BCC & 10.19 & 10.53 & 3.35 \\
\hline \multirow{4}{*}{ Diesel HEV } & HTUF & 10.00 & 10.05 & 0.46 \\
\cline { 2 - 5 } & HHDDT & 10.50 & 10.92 & 4.00 \\
\cline { 2 - 5 } & NY COMP & 8.81 & 8.66 & -1.70 \\
\hline & & & & \\
\hline
\end{tabular}

\section{Statistics of Driving Trip Characteristics}

More than 1,400 vehicle-day data were collected from MD parcel delivery trucks. After removing vehicle-days with fewer than five miles or more than 150 miles of driving, this study used the remaining 1,290 vehicle-days to support the analysis.
The FC is highly dependent on driving profile properties like vehicle speed, kinetic intensity (KI) [11], and travel distance. For a given trip, it is important to use the average speed and KI when analyzing the FC. The average speeds and KI of real-world trips imply the extent to which the trips are transient. Low-speed or high-KI trips are more transient. Both the idle and stop-start behavior are more common at low speeds or high KI modes than on the highway. The statistics of the driving trip properties are shown in Figures 2 through $\underline{4}$ It should be noted that the idle time was included when computing the average speed of a trip in this study. From these figures we can see that over $90 \%$ of the trips have a KI ranging from 1 to 5 , an average speed less than $25 \mathrm{mph}$, and a daily travel distance less than 75 miles, which will be applied in the following analysis.

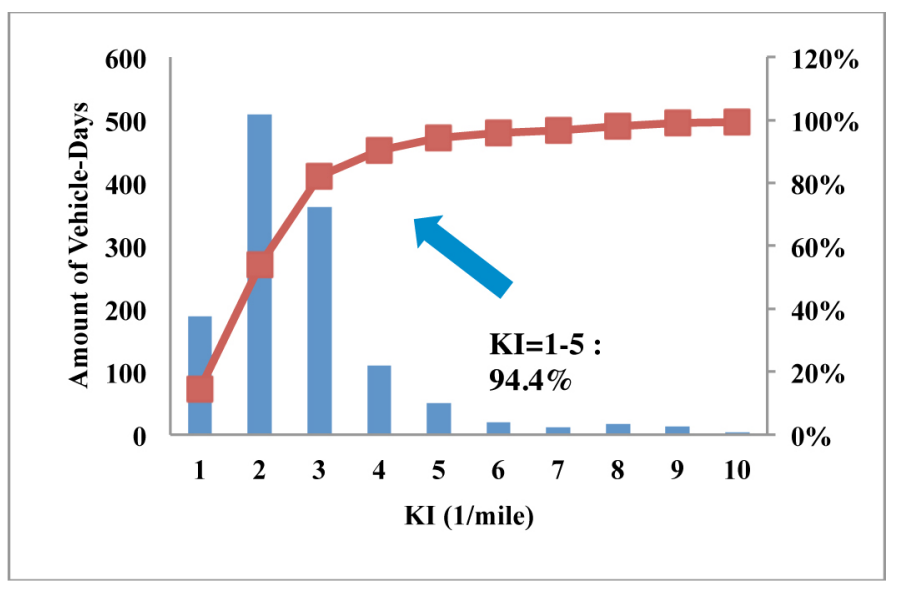

Figure 2. Trip KI

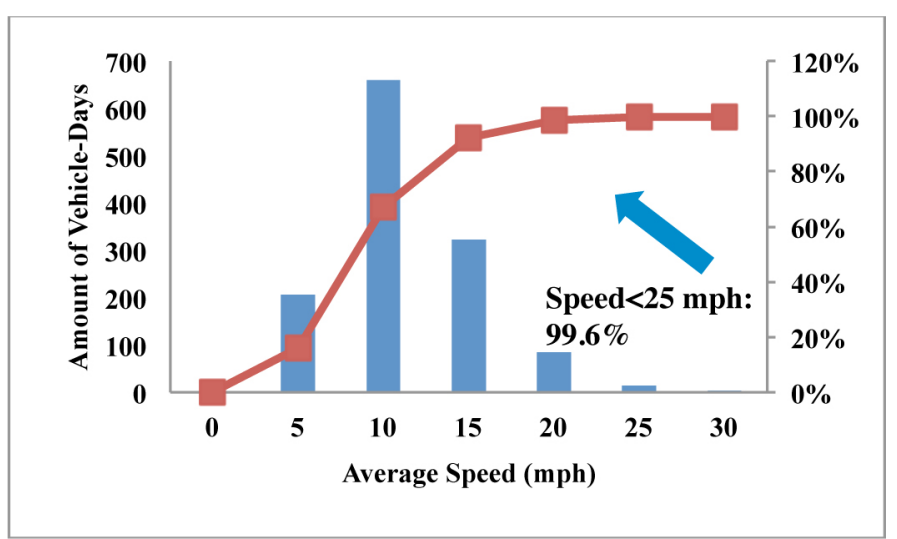

Figure 3. Trip average speed

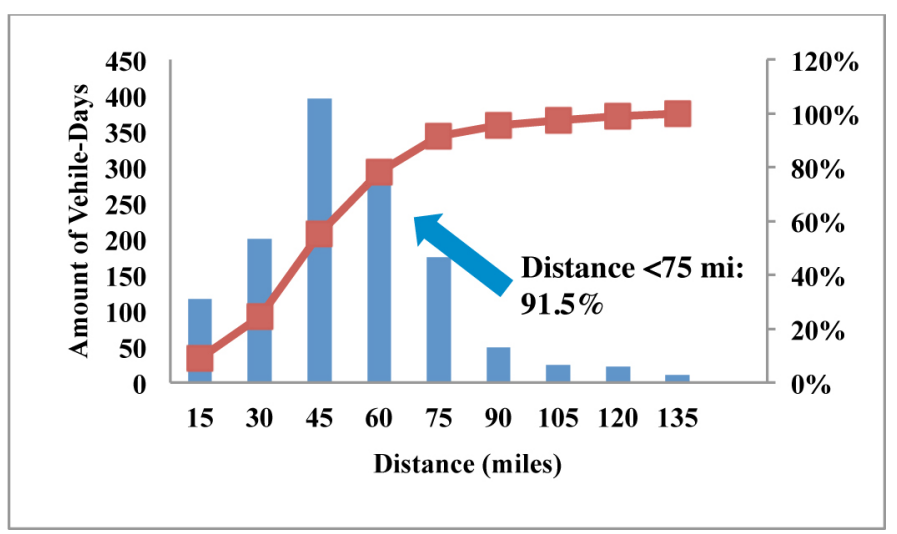

Figure 4. Trip daily travel distance 


\section{Impact of Parametric Reduction on Fuel Consumption}

\section{Rolling Resistance Reduction}

Tire rolling resistance is proportional to the product of the coefficient of rolling resistance, vehicle mass, and gravity acceleration, which accounts for around $30 \%$ of the vehicle's resistance to forward motion [12]. The conventional diesel parcel delivery truck was simulated on FASTSim over 1,290 real-world trips by changing the rolling resistance at a constant weight and aerodynamic drag. Figure $\underline{5}$ shows the impacts of rolling resistance reduction and average speed on FC over 1,290 trips at a constant test weight and aerodynamic drag. The impacts of rolling resistance reduction and $\mathrm{KI}$ on fuel saving are depicted in Figure 6. Clear trend lines are demonstrated on both plots, which means both average speed and KI can be used for a study of trip characteristics. It also can be seen that $0.5 \%-1.6 \%$ fuel saving would be achieved with a reduction of $10 \%$ in rolling resistance, depending on the trips.

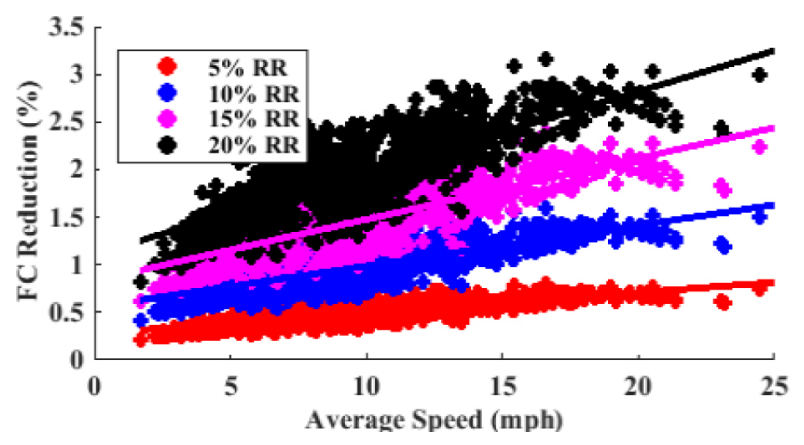

Figure 5. Impacts of rolling resistance reduction and average speed on FC reduction

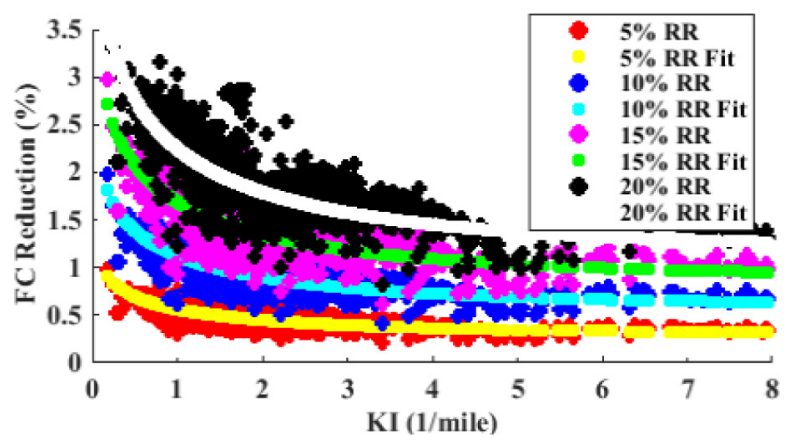

Figure 6. Impacts of rolling resistance reduction and $\mathrm{KI}$ on FC reduction

The daily fuel savings (in gallons) for the 1,290 vehicle-days were calculated using equation 1.

$$
\text { daySaving = dayFuelSavingRate } * \text { dayVMT }
$$

where dayFuelSavingRate is the daily fuel savings per mile and dayVMT is the 1,290 vehicle daily trips. Figure 7 shows the daily fuel savings when the rolling resistance is reduced by $5 \%$ to $20 \%$. Total daily fuel savings of 1,290 vehicle-days are also displayed on the plot.

Assuming the parcel delivery trucks are used 5 days a week for 50 weeks a year, 1,290 vehicle-days equal 5.15 vehicle-years. The lifetime fuel saving would be 256 gallons, assuming a vehicle life of
15 years. The cost effectiveness of the upfront cost is less than $\$ 921$ to reduce rolling resistance by $10 \%$, assuming a $\$ 3.60$ per gallon projected diesel price [13].

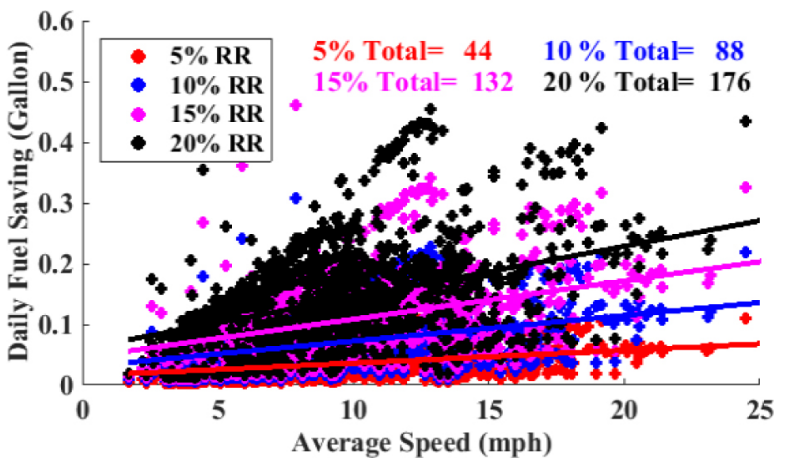

Figure 7. Daily fuel savings with reduced rolling resistance

Incremental rollout of adopting lower rolling resistance was investigated, beginning with the most favorable driving routes. Figures $\underline{8}$ and $\underline{9}$ show the distributions of probability and cumulative percentage of daily fuel saving when reducing rolling resistance by $5 \%$ and $10 \%$.

The areas to the right of the vertical lines achieve more fuel saving than the total average fuel saving shown in Figure 8 . Figure 9 shows that $40 \%$ of the total trips will achieve more than average daily fuel savings. Figures 10 and $\underline{11}$ show daily fuel savings versus the characteristics of driving profiles, which depicts the most favorable trips on which to adopt the new technology. This analysis suggested that the parcel delivery fleet might need to first install equipment to reduce the rolling resistance on trucks driving long distance, low-KI, high-speed trips.

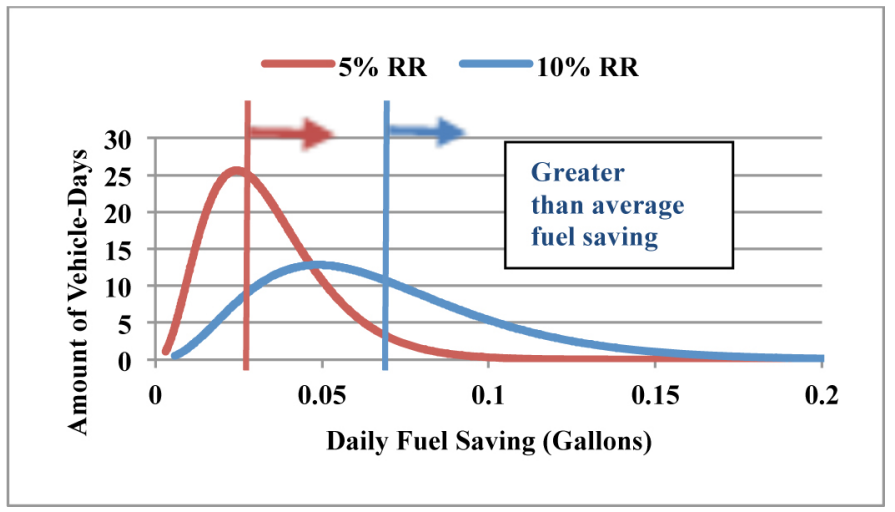

Figure 8. Probability distribution of daily fuel saving with reduction in rolling resistance

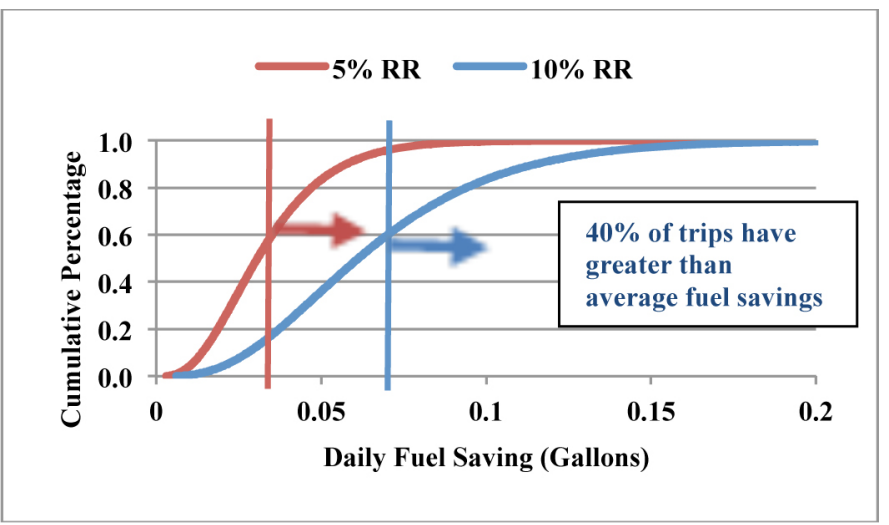

Figure 9. Cumulative percentage distribution of daily fuel saving with reduction in rolling resistance 


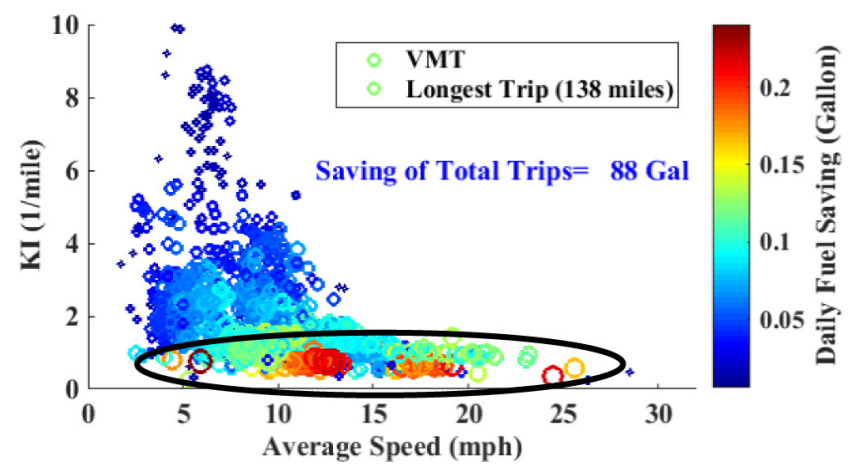

Figure 10. Daily fuel savings vs. characteristics (average speed and KI) of driving trips with $10 \%$ reduction in rolling resistance

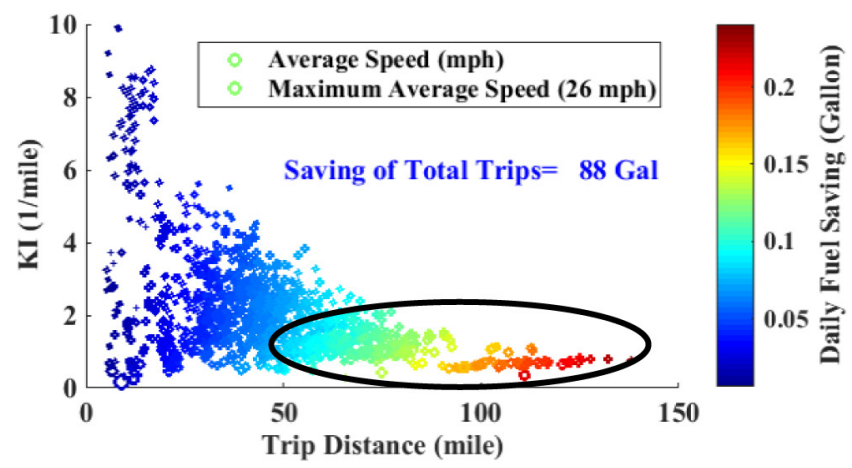

Figure 11. Daily fuel savings vs. characteristics (trip distance and KI) of driving trips with $10 \%$ reduction in rolling resistance

\section{Aerodynamic Drag}

The metric for evaluating aerodynamic losses is aerodynamic drag which is a force opposing the motion of the vehicle caused by the resistance of ambient air. Quantitatively, the aerodynamic drag is proportional to the product of the coefficient of aerodynamic drag, the frontal area, and the square of vehicle velocity. Reducing the frontal area usually sacrifices interior size and thus has limited value in reducing aerodynamic drag. Driving slower can also reduce the drag force. However, changing the actual road speed is not realistic, so reducing the drag coefficient is the main way to reduce aerodynamic drag. The conventional diesel parcel delivery truck was simulated on FASTSim over 1,290 real-world trips by changing aerodynamic drag at a constant weight and rolling resistance. Figure 12 shows the impacts of aerodynamic drag reduction and average speed on FC reduction over 1,290 vehicle-day trips. It can be seen that $0.5 \%-5 \%$ fuel saving would be achieved with a $10 \%$ aerodynamic drag reduction, depending on the trips.

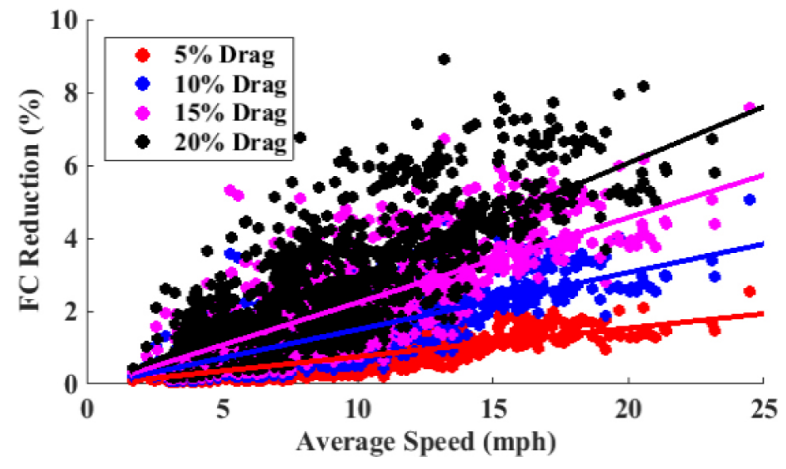

Figure 12. Impacts of aerodynamic drag reduction and average speed on FC reduction
The daily fuel savings due to aerodynamic drag reduction are demonstrated in Figure 13. Fuel savings for 1,290 total trips are displayed on the plot. Assuming the parcel delivery trucks are driven 5 days a week for 50 weeks a year, 1,290 vehicle-days equal 5.15 vehicle-years. The lifetime fuel saving would be 396 gallons, assuming a vehicle life of 15 years. The cost effectiveness of the upfront cost is less than $\$ 1,426$ to reduce aerodynamic drag by $10 \%$, assuming a $\$ 3.60$ per gallon projected diesel price [13] .

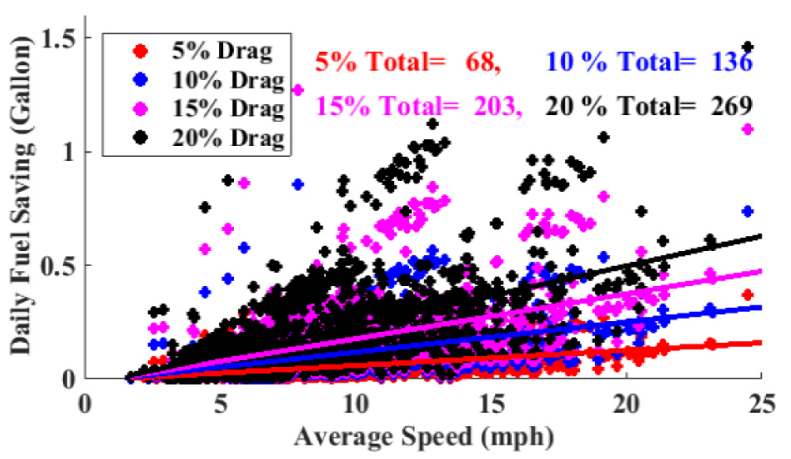

Figure 13. Daily fuel savings with reduced aerodynamic drag

The most favorable driving routes on which to adopt low aerodynamic drag technologies were also inspected. Figure 14 shows the distribution of cumulative percentage of daily fuel savings when reducing aerodynamic drag by $5 \%$ and $10 \%$, from which we can see that $32 \%$ of the total trips achieve a greater-than-average daily fuel savings. Detailed information on fuel savings versus the characteristics of driving profiles is demonstrated in Figures 15 and 16. Trips with high speed, long distance, and low KI are recommended to adopt new technologies to reduce daily fuel savings.

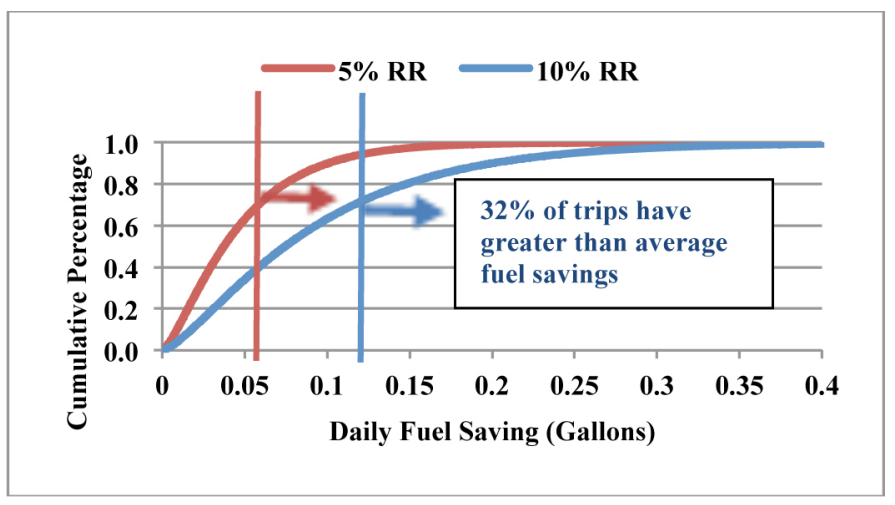

Figure 14. Cumulative percentage distribution of daily fuel saving with reduced aerodynamic drag

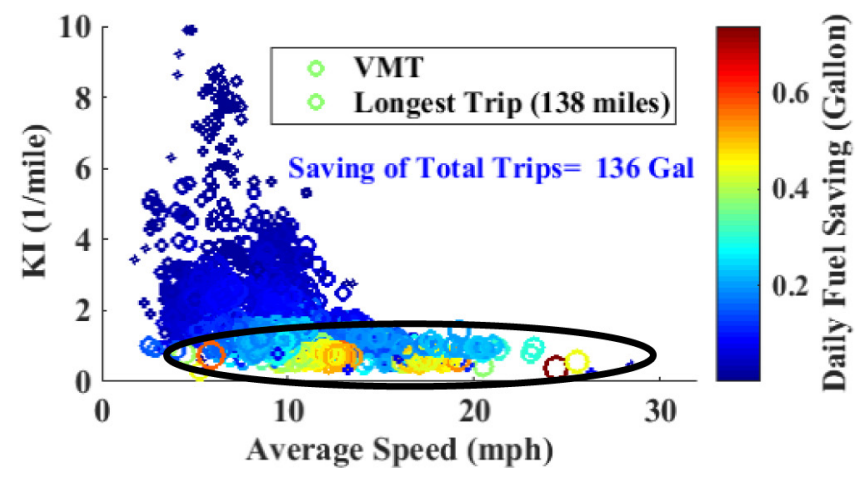

Figure 15. Daily fuel savings vs. characteristics (average speed and KI) for driving trips with $10 \%$ aerodynamic drag reduction 


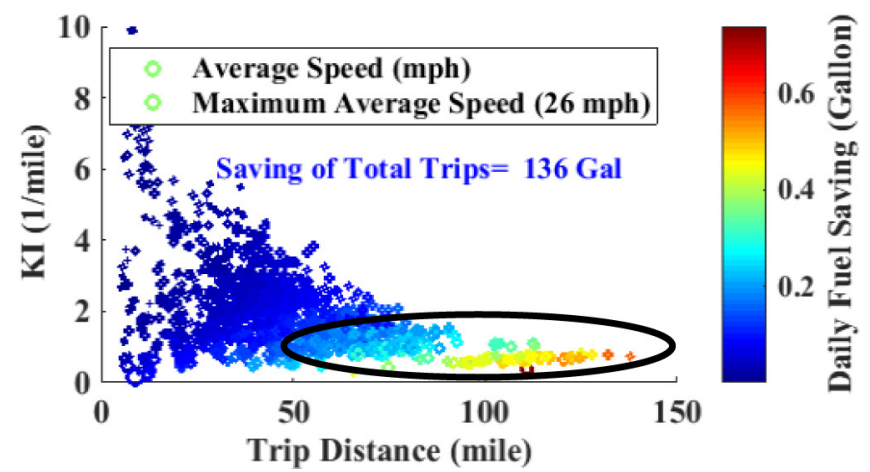

Figure 16. Daily fuel savings vs. characteristics (trip distance and KI) of driving trips with $10 \%$ aerodynamic drag reduction

\section{Vehicle Weight}

The vehicle weight affects the engine power required to propel the vehicle through acceleration, rolling resistance, and hill climbing. Reducing the vehicle's weight could either save fuel or increase the freight carried. The parcel delivery truck was simulated in FASTSim by reducing the curb weight by $5 \%$ to $20 \%$ over 1,290 real-world trips at a constant aerodynamic drag and rolling resistance.

Figure 17 shows the impacts of weight reduction and average speed on FC over 1,290 day trips. An FC reduction up to $6 \%$ was achieved when the curb weight was reduced by $10 \%$. There would be more impact in the real world if grade information were included. The daily fuel savings with weight reductions are demonstrated in Figure 18 , with the 1,290 total trips savings displayed on the plot.

Assuming the parcel delivery trucks are driven 5 days a week for 50 weeks a year, 1,290 vehicle-days equal 5.15 vehicle-years. The lifetime fuel saving would be 1,016 gallons, assuming a vehicle life of 15 years. The cost effectiveness of the upfront cost is less than $\$ 3,659$ to reduce vehicle mass by $10 \%$ assuming a $\$ 3.60$ per gallon projected diesel price [13]

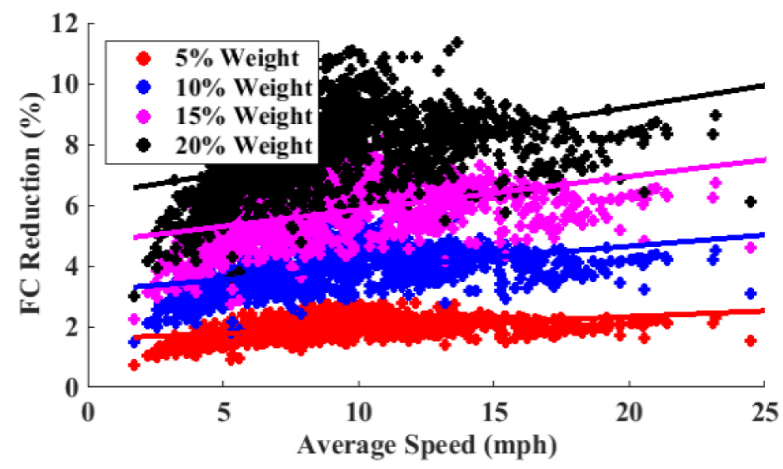

Figure 17. Impacts of aerodynamic weight reduction and average speed on FC

Similarly, the most favorable driving routes on which to adopt lightweight material vehicles were researched. The distribution of cumulative percentage of daily fuel savings when reducing aerodynamic drag by $5 \%$ and $10 \%$ is shown in Figure 19 . A total of $49 \%$ of the trips achieve greater-than-average daily fuel saving. Figures 20 and 21 demonstrate the detailed information of fuel savings versus the characteristics of driving profiles. The vehicle speed and KI are not the only key factors that determine the daily fuel savings because the weight affects FC through acceleration and rolling resistance. Trips traveled at large speed over a long distance with large acceleration should be good candidates on which to use lighter-weight trucks.

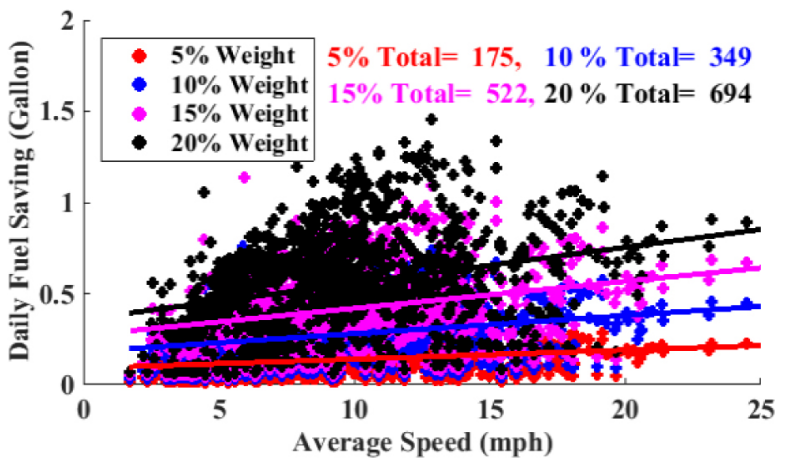

Figure 18. Daily fuel savings with reduction in weight

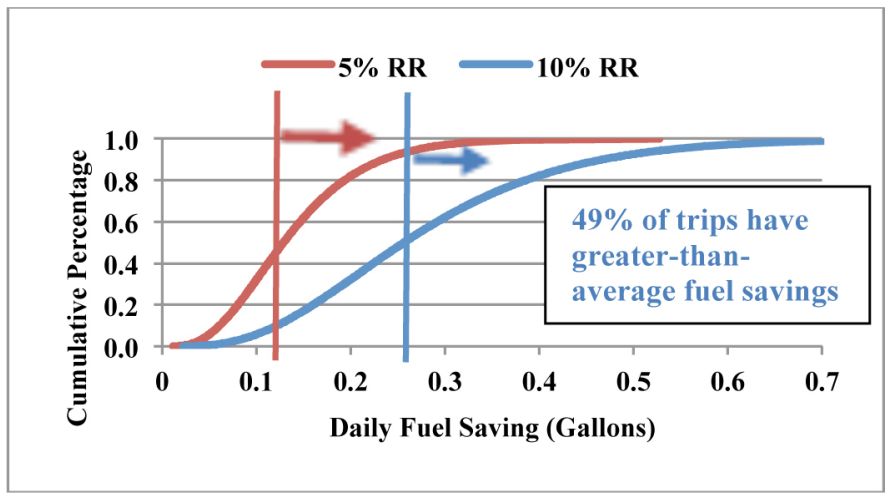

Figure 19. Cumulative percentage distribution of daily fuel saving with reduced vehicle weight

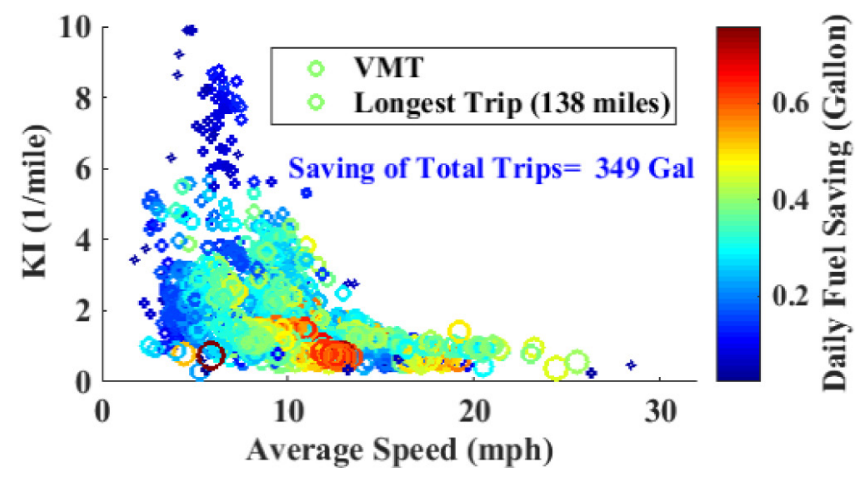

Figure 20. Daily fuel savings vs. characteristics (average speed and KI) of driving trips with $10 \%$ weight reduction

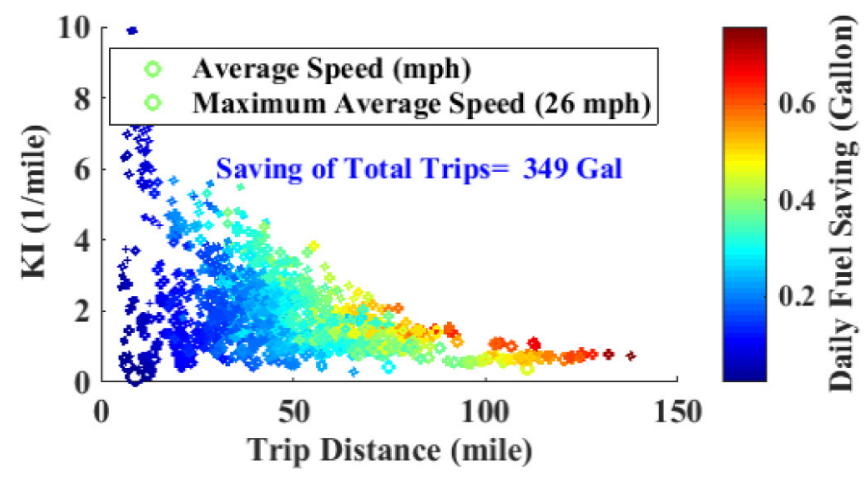

Figure 21. Daily fuel savings vs. characteristics (average speed and KI) of driving trips with $10 \%$ weight reduction 


\section{Daily Fuel Saving Comparison among Three Technologies}

As seen above, the daily fuel savings significantly differ when different technologies are examined. Figures 22 and 23 show the distributions of probability and cumulative percentage of daily fuel savings when rolling resistance, aerodynamic drag, and weight are reduced by $10 \%$. Overall, with the same amount of technology reduction, the fuel savings are largest for a reduction in weight and least with a reduction in rolling resistance, which is consistent with our observations. It should be noted that fuel savings due to reduced rolling resistance are larger than with same amount of reduction in the aerodynamic drag at low speeds.

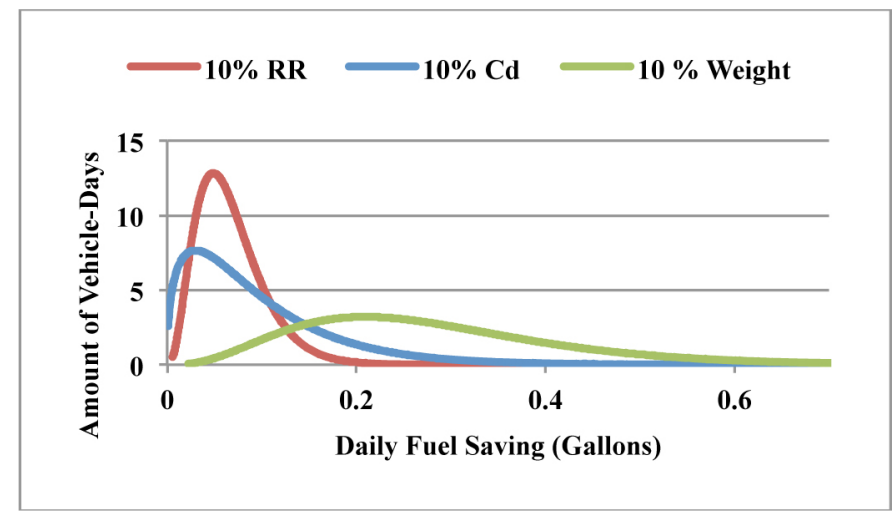

Figure 22. Probability distribution of daily fuel savings with $10 \%$ reduction in rolling resistance, aerodynamic drag, and weight

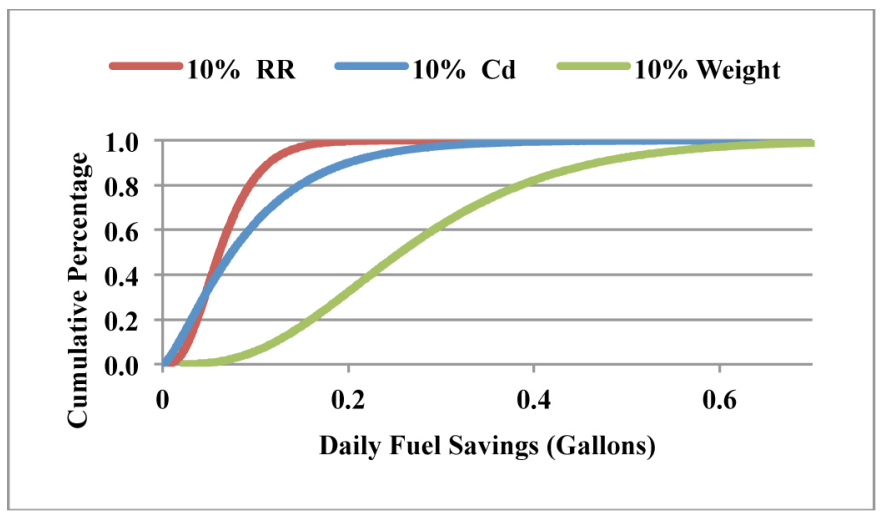

Figure 23. Cumulative percentage distribution of daily fuel savings with a $10 \%$ reduction in rolling resistance, aerodynamic drag, and weight

\section{Hybridization}

The use of HEVs allows truck manufacturers to simultaneously improve fuel efficiency and performance. Furthermore, HEV technology could provide a technological and commercial bridge from today's conventional powertrains for future fuel cell powertrains [14]. HHVs use hydraulic pumps and motors with low-pressure and high-pressure reservoirs to absorb and deliver torque from the drivetrain, which is also practical for commercial truck applications. Both HEV and HHV save fuel because of the following:

- Optimum engine operating region: The engine operates closely to its best fuel efficiency line.
- Engine shut off: Fuel efficiency is very low when the engine operates at a low speed. The engine is shut off when its speed is below a certain threshold to save fuel.

- $\quad$ Regenerative braking: A regenerative brake recovers the kinetic energy produced when braking into electrical energy which can be stored in the energy storage system for future use.

Three trucks with the same specifications (as shown in Table 1) are used to support this analysis. The baseline truck is a conventional gasoline parcel delivery truck, and the two hybrid trucks are an HEV and an HHV. The three trucks were simulated in FASTSim over 1,290 real world trips. The daily fuel savings with hybridization are demonstrated in Figure 24. The total fuel savings over the 1,290 vehicle-days are displayed on the plot.

Assuming the parcel delivery trucks are driven 5 days a week for 50 weeks a year, 1,290 vehicle-days equal 5.15 vehicle-years. The lifetime fuel savings would be 11,377 gallons and 10,509 gallons for HEV and HHV, respectively, assuming a vehicle life of 15 years. The cost effectiveness of the upfront costs is less than $\$ 40,956$ for the HEV and $\$ 37,831$ for the HHV to reduce vehicle mass by $10 \%$, assuming a $\$ 3.60$ per gallon projected diesel price [13] .

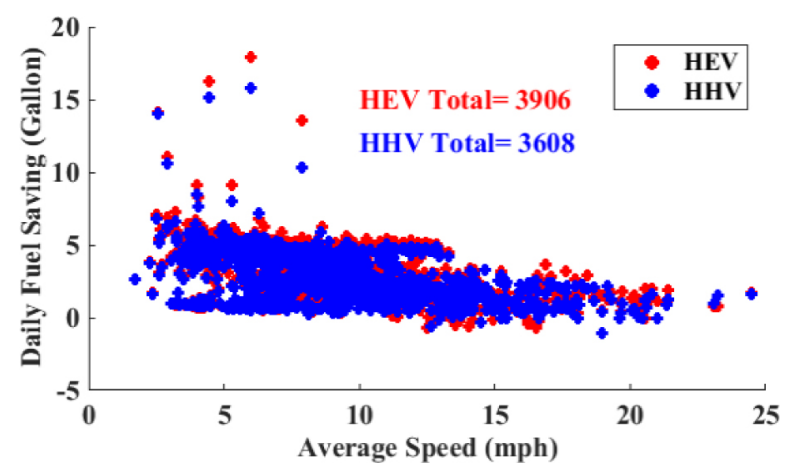

Figure 24. Daily fuel savings with hybridization (HEV and HHV)

Likewise, the most favorable driving routes on which to use hybridized parcel delivery trucks were investigated. Figure 25 shows the distribution of cumulative percentage of daily fuel savings when replacing a conventional truck with an HEV or HHV. As observed above, HHVs and HEVs share similar fuel saving behaviors in which approximately $44 \%$ of the total trips achieve greater-than-average daily time fuel saving. The detailed information for fuel savings due to hybridization versus the characteristics of the driving profiles is demonstrated in Figures 26 and $\underline{27}$. It should be noted that only the HEV scenarios are shown in the plots since HEVs and HEVs share similar fuel saving behavior. From these figures, we can see that trucks traveling on trips with lower average speeds and high KIs have the largest benefit because the truck has more energy recovery in stop-andgo scenarios. Unlike with reductions in rolling resistance, aerodynamic drag, and weight, the trip distance are not the key factors that determine the fuel saving with hybridization because a long travel distance normally means that trucks travel most of the time on highways. 


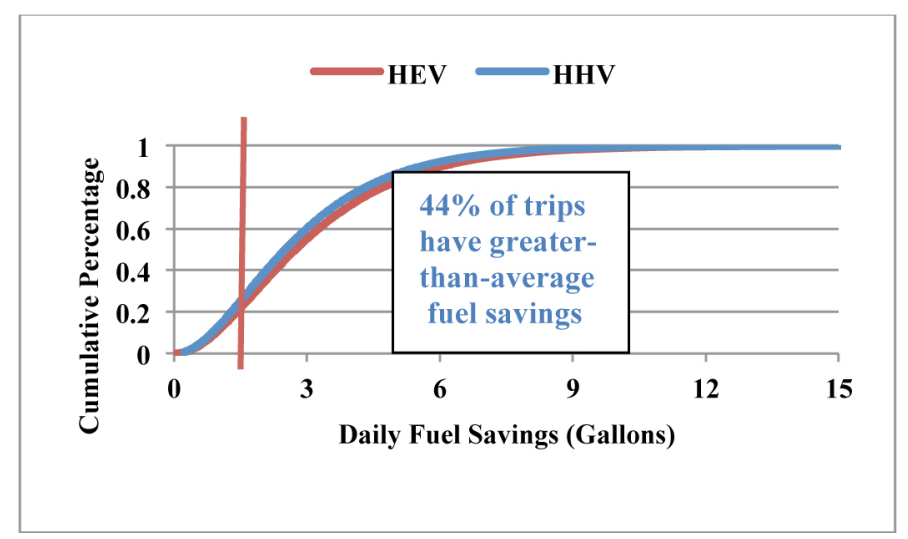

Figure 25. Cumulative percentage distribution of daily fuel savings with hybridization for HEVs and HHVs

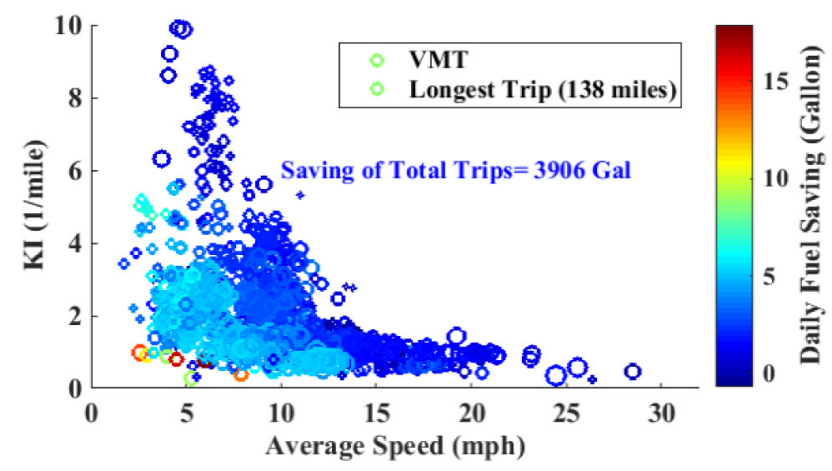

Figure 26. Daily fuel savings vs. characteristics (average speed and KI) of driving trips with hybridization

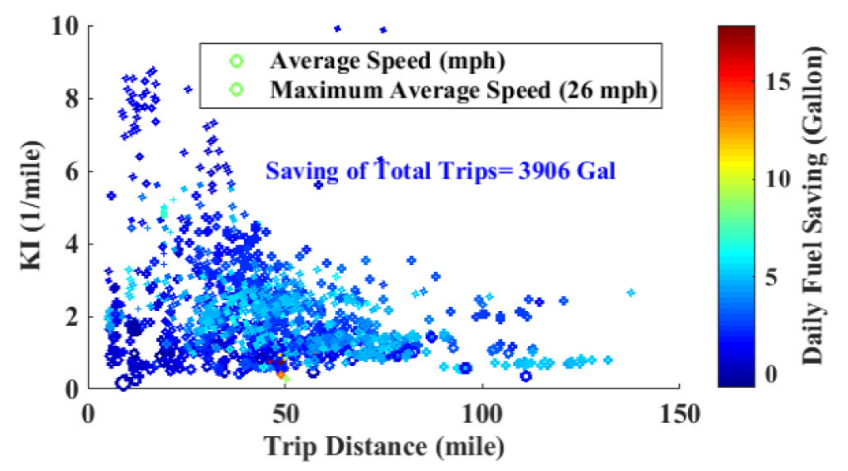

Figure 27. Daily fuel savings vs. characteristics (trip distance and KI) of driving trips with hybridization

\section{Summary}

The impacts of reductions in rolling resistance, aerodynamic drag, and weight, as well as hybridization of the vehicles on fuel savings were investigated, and the most favorable driving routes on which to adopt new technologies were suggested by simulating parcel delivery trucks using FASTSim over 1,290 real-world driving trips. The study revealed that reductions up to $2 \%, 4 \%$, and $6 \%$ in FC were achieved when reducing rolling resistance, aerodynamic drag, and curb weight by $10 \%$, respectively, depending on the characteristics of the driving trips. Overall, with an average of over 12,000 gallons fuel saved daily, the fuel savings due to hybridization surpassed that of reductions in rolling resistance, aerodynamic drag, and weight. This study proposed that, if the cost of new technologies is known, depending on the circumstances it may be more cost effective to adopt one technology to save fuel, or it may be more beneficial to adopt another.

\section{References}

1. Brooker, A., Gonder, J., Wang, L., Wood, E. et al., "FASTSim: A Model to Estimate Vehicle Efficiency, Cost and Performance," SAE Technical Paper 2015-01-0973, 2015, doi:10.4271/201501-0973.

2. Burton, J., Walkowicz, K., Sindler, P., and Duran, A., "InUse and Vehicle Dynamometer Evaluation and Comparison of Class 7 Hybrid Electric and Conventional Diesel Delivery Trucks," SAE Int. J. Commer. Veh. 6(2):545-554, 2013, doi: 10.4271/2013-01-2468.

3. Lammert, M., Walkowicz, K., Duran, A., and Sindler, P., "Measured Laboratory and In-Use Fuel Economy Observed over Targeted Drive Cycles for Comparable Hybrid and Conventional Package Delivery Vehicles," SAE Technical Paper 2012-01-2049, 2012, doi:10.4271/2012-01-2049.

4. Lammert, M., Burton, J., Sindler, P., and Duran, A., "Hydraulic Hybrid and Conventional Parcel Delivery Vehicles' Measured Laboratory Fuel Economy on Targeted Drive Cycles," SAE Int. J. Alt. Power. 4(1):11-19, 2015, doi:10.4271/2014-01-2375.

5. Barnitt, R., FedEx Express Gasoline Hybrid Electric Delivery Truck Evaluation: 12-Month Report. Golden, CO: National Renewable Energy Laboratory. NREL Technical Report TP5400-48896, 2011.

6. Lammert, M., Walkowicz, K., Thirty-Six Month Evaluation of UPS Diesel Hybrid-Electric Delivery Vans. Golden, CO: National Renewable Energy Laboratory. NREL Technical Report TP-5400-53503, 2012.

7. Lammert, M., Walkowicz, K., Eighteen-Month Final Evaluation of UPS Second Generation Diesel Hybrid-Electric Delivery Vans, Golden, CO: National Renewable Energy Laboratory. NREL Technical Report TP-5400-55658, 2012.

8. Lammert, M., Burton, J., Sindler, P., and Duran, A., "Hydraulic Hybrid and Conventional Parcel Delivery Vehicles' Measured Laboratory Fuel Economy on Targeted Drive Cycles," SAE Int. J. Alt. Power. 4(1):11-19, 2015, doi:10.4271/2014-01-2375.

9. Gallo, J., Tomic, J., "Battery Electric Parcel Delivery Truck Testing and Demonstration," California Hybrid, Efficient and Advanced Truck Research Center (CALSTART). 2013.

10. Wang, L., Duran, A., Gonder, J., and Kelly, K., "Modeling Heavy/Medium-Duty Fuel Consumption Based on Drive Cycle Properties," SAE Technical Paper 2015-01-2812, 2015, doi:10.4271/2015-01-2812.

11. O'Keefe, M., Simpson, A. and Kelly, K., "Duty Cycle Characterization and Evaluation Towards Heavy Hybrid Vehicle Applications," Presented at the 2007 SAE World Congress and Exhibition, 16-19 April 2007, Detroit, Michigan. Golden, CO: National Renewable Energy Laboratory. NREL/CP-540-40929.

12. Clark, N., Gautam, M., Wayne, W., Nine, R. et al., "Creation and Evaluation of a Medium Heavy-Duty Truck Test Cycle," SAE Technical Paper 2003-01-3284, 2003, doi:10.4271/200301-3284.

13. U.S. Energy Information Administration, "Annual Energy Outlook 2010 with Projections to 2035,” DOE/EIA-0383(2010), 2010. http://www.eia.gov/oiaf/aeo/pdf/0383(2010).pdf, accessed April 12, 2016. 
14. $21^{\text {st }}$ Century Truck Partnership, "Roadmap and Technical White Papers." Final - February 27, 2013. https://www1.eere.energy. gov/vehiclesandfuels/pdfs/program/21ctp_roadmap_2007.pdf

\section{Contact Information}

Lijuan Wang

Lijuan.wang@nrel.gov

\section{Acknowledgments}

This work was supported by the U.S. Department of Energy under Contract No. DE-AC36-08GO28308 with the National Renewable Energy Laboratory. Funding was provided by U.S. DOE Office of Energy Efficiency and Renewable Energy Vehicle Technologies Office. The U.S. Government retains and the publisher, by accepting the article for publication, acknowledges that the U.S. Government retains a nonexclusive, paid-up, irrevocable, worldwide license to publish or reproduce the published form of this work, or allow others to do so, for U.S. Government purposes.

\section{Definitions/Abbreviations}

BCC - Baltimore Custom Cycle

CSHVC - City Suburban Heavy Vehicle Cycle

FASTSim - Future Automotive Systems Technology Simulator

FC - fuel consumption

HEV - hybrid electric vehicle

HHDDT - Heavy Heavy-Duty Diesel Truck

HHV - hydraulic electric vehicle

HTUF - Hybrid Truck Utility Forum Class 4

KI - kinetic intensity

MD - medium duty

NREL - National Renewable Energy Laboratory

NY COMP - New York City Composite

ReFUEL - Renewable Fuels and Lubricants

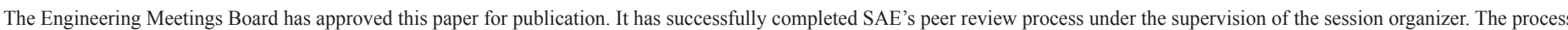
requires a minimum of three (3) reviews by industry experts.

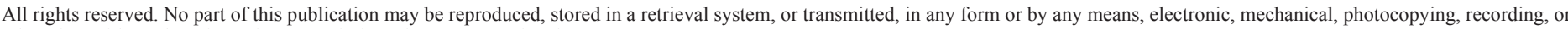
otherwise, without the prior written permission of SAE International.

Positions and opinions advanced in this paper are those of the author(s) and not necessarily those of SAE International. The author is solely responsible for the content of the paper.

ISSN 0148-7191

http://papers.sae.org/2016-01-8134 